Schmerz 2013 $27: 454-455$

DOI 10.1007/s00482-013-1355-5

Online publiziert: 15 . September 2013

(c) Deutsche Schmerzgesellschaft e.V.

Published by Springer-Verlag Berlin Heidelberg -

all rights reserved 2013

\author{
M. Dusch ${ }^{1} \cdot$ C. Quandt ${ }^{2}$ \\ ${ }^{1}$ Klinik für Anästhesiologie und Operative Intensivmedizin, Medizinische Fakultät Mannheim \\ der Universität Heidelberg, Mannheim \\ ${ }^{2}$ Klinik für Anästhesiologie und Intensivmedizin, Medizinische Hochschule Hannover
}

\title{
Lehre mit Schmerz
}

Mit der Novelle der Approbationsordnung im Juli 2012 wurde die Einführung eines neuen Querschnittsbereichs 14 „Schmerzmedizin“ beschlossen. Alle medizinischen Fakultäten haben daher die Arbeit an einen Curriculum aufgenommen oder werden dies in Kürze tun. Der entsprechende Leistungsnachweis wird ab Oktober 2016 verbindlich für die Anmeldung zum Staatsexamen. In diesem Beitrag möchten wir die Vielfalt der möglichen Lösungen, generelle Überlegungen zur Unterrichtsgestaltung sowie die Konsequenzen für den zeitlichen Ablauf der curricularen Implementierung beleuchten.

One size fits all?! - One size fits none!

\section{Individuelle Lösungen}

Bereits im Jahr 2008 hat die Deutsche Schmerzgesellschaft ein 15 Unterrichtseinheiten umfassendes Mustercurriculum für den Querschnittsbereich Schmerzmedizin veröffentlicht (http://www.dgss. org). Diese kann als eine inhaltliche Leitlinie für die lokale Implementierung dienen - erste Erfahrungen mit einer erfolgreichen Implementierung wurden bereits publiziert. Ziel des Mustercurriculums ist die Vermittlung des Kernwissens sowie der Kernkompetenzen für die Erkennung und Behandlung von Schmerzen durch den zur Weiterbildung befähigten Arzt. Die Autoren legen explizit Wert auf eine Abgrenzung hin zur gebietsbezogenen Zusatzbezeichnung „Spezielle Schmerztherapie" und unterstreichen so die Relevanz der curricularen Inhalte für die all- gemeine ärztliche Tätigkeit. Die Verbreitung dieses Mustercurriculums bietet die große Chance zu einer Intensivierung der Zusammenarbeit der medizinischen Fakultäten auf diesem Gebiet.

Durch die Vielfalt der Regel-, Reformund Modellstudiengänge wird eine Betrachtung des Curriculums aus ganz unterschiedlichen Blickwinkeln ermöglicht und so, ein strukturiertes Feedback voraus gesetzt, die weitere Evolution auf didaktischer und inhaltlicher Ebene vorangetrieben. Im Sinne der Ressourcenschonung kann so auch die Doppelung von Arbeit vermieden werden. Als Plattform für diese Curriculumverbreitung bietet die Deutsche Schmerzgesellschaft seit dem Frühjahr 2013 Q14-Workshops an. Die Vielfalt der unterschiedlichen Studiengänge und lokalen Besonderheiten macht einen einheitlichen Lösungsansatz für die curriculare Implementierung unmöglich. Vielmehr sind individuelle Lösungen gefragt! Das dafür nötige Werkzeug der Curriculumsentwicklung bietet der sechsstufige Ansatz von Kern [1].

\section{Methodisches Alignment}

Damit Lehren und Lernen gelingen können, sollten Lernziele, Unterrichtsmethoden und Prüfungsaufgaben aufeinander abgestimmt sein. Nach Biggs u. Tang [2] wird unter "constructive alignment“ verstanden, dass Unterrichts- und Lerneraktivitäten sowie die Prüfungsaufgaben sich direkt auf die zu erreichenden Lernziele beziehen. Dieses auf kognitiver Psychologie und Biologie beruhende Konzept hat zwei zugrunde liegende Prinzipien:
Lernende bilden für sich selbst und individuell eine Bedeutung aus dem Gelernten, in dem sie es mit Vorwissen und Erfahrungen verknüpfen, grundlegende Prinzipien anwenden und durch Extrapolation auf zukünftige Ereignisse schließen. Wir wenden also verschiedene Filter an, um unsere chaotische Realität zu ordnen und das Wissen so anzulegen, dass es in unsere Welt passt.

Lehrende richten die Unterrichtsaktivitäten und die Lernziele aufeinander aus. Damit bekommen die Lernenden klar definierte Lernziele, zu diesen Zielen passende Unterrichtsmethoden und darauf ausgelegte Prüfungskriterien mit dem entsprechenden Feedback. Unterrichtsmethoden, die das spielerische Entdecken und Experimentieren oder gemeinschaftliches und projektbezogenes Lernen fördern, spielen eine besondere Rolle.

\section{Roadmap to 2016}

Unabhängig von der Tatsache, ob es den Fakultäten gelingt, durch Änderung des Stundenplans Zeiten für Blockunterricht zu schaffen oder ob ein longitudinales Konzept entwickelt wird, wird durch die Änderung der ärztlichen Approbationsordnung der zeitliche Ablauf vorgegeben: Alle Studierenden, die im Oktober 2016 das Praktische Jahr beginnen wollen, müssen in Schmerzmedizin geprüft worden sein. Bis 2014 sollte ein entsprechendes Curriculum entwickelt sein, damit dieses zeitgerecht die Sitzungen der entsprechenden Gremien wie Studienoder Curriculumskommission sowie Prüfungsausschuss passieren kann. Erste Prüfungen sollten sinnvollerweise bis 2015 
stattfinden, damit evtl. noch Anpassungen vorgenommen werden können.

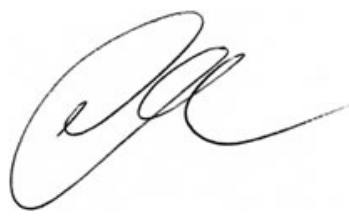

M. Dusch

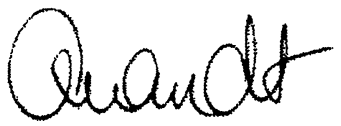

C. Quandt

\section{Korrespondenzadresse}

\section{Dr. M. Dusch}

Klinik für Anästhesiologie und Operative Intensivmedizin,

Medizinische Fakultät Mannheim der Universität Heidelberg, Mannheim Theodor-Kutzer-Ufer 1-3, 68167 Mannheim martin.dusch@umm.de

\section{Einhaltung ethischer Richtlinien}

Interessenkonflikt. M. Dusch und C. Quandt Taylor geben an, dass kein Interessenkonflikt besteht.

Dieser Beitrag beinhaltet keine Studien an Menschen oder Tieren.

\section{Literatur}

1. Kern DE, Thomas PA, Howard DM, Bass EB (1998) Curriculum development for medical education: a six-step approach. John Hopkins University Press, Baltimore London

2. Biggs J, Tang C (2011) Teaching for quality learning at university. McGraw-Hill and Open University Press, Maidenhead

\section{Boris Zernikow (Hrsg.) \\ Palliativversorgung von Kindern, Jugendlichen und jungen Erwachsenen}

Heidelberg: Springer-Verlag GmbH 2013,

2., überarb. Auflage, $565 \mathrm{~S}$.,

(ISBN 978-3-642-29609-3), 79.95 EUR

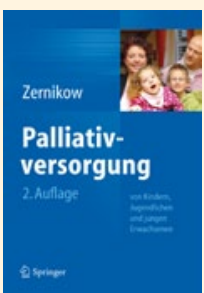

Fünf Jahre nach Erstauflage erscheint nun die komplett überarbeitete und ergänzte 2. Auflage. Das Buch ist in drei große Abschnitte gegliedert: Abschnitt 1 beschäftigt sich mit den strukturel-

len, organisatorischen und ethischen Grundlagen der Kinderpalliativversorgung.

Abschnitt 2, das Herzstück des Buches, widmet sich der Praxis der pädiatrischen Palliativversorgung und behandelt Themen wie Todeskonzepte von Kindern, praktische Schmerztherapie, Symptomlinderung von belastender körperlicher Symptome wie spezifische Krankheitsbilder der Kinderpalliativversorgung.

Im 3. Abschnitt geht es um die Familien und um die Versorger. Darüber hinaus werden rechtliche Aspekte diskutiert.

Aus-, Fort- und Weiterbildungsmöglichkeiten sowie ein Ausblick in den Bereich der Forschung runden das Buch ab.

Das Buch bietet insgesamt einen exzellenten Überblick über die Thematik. Die wichtigen Aspekte praktischer Symptomkontrolle werden detailreich und mit vielen Beispielen plastisch und verständlich dargestellt. Es werden "nachkochbare Rezepte" angeboten und es wird intensiv über medikamentöse Behandlungsmöglichkeiten berichtet. Darüber hinaus werden aber auch nicht medikamentöse Maßnahmen vorgestellt und wichtige Hinweise und Handlungsvorschläge für die Bereiche Ethik und Recht gegeben. Insgesamt lässt uns das Autorenteam an ihrem Erfahrungsschatz teilhaben und gibt uns eine Vielzahl praktischer Tipps an die Hand, die im eigenen beruflichen Alltag umgesetzt werden können. So stellt das Buch eine gelungen Mischung aus Nachschlagewerk und Praxisleitfaden dar, der sowohl Ärzte als auch Pflegende sowie alle weiteren Versorger lebenslimitierend erkrankter Kinder, Jugendlicher und junger Erwachsener in gleicher Weise anspricht. Das Buch ist zudem so gut lesbar und verständ- lich geschrieben dass es tatsächlich auch für Eltern betroffener Kinder geeignet scheint, um sich zumindest in einzelnen Bereichen tiefergehend zu informieren. Insgesamt ein rundum gelungenes Buch, in seiner Art einzigartig und somit ohnehin ein Pflichtkauf für jeden auf diesem Gebiet Tätigen.

PD Dr. Sven Gottschling (Homburg/Saar) 